\title{
Characteristics of patients with heart failure with preserved ejection fraction in primary care: a cross-sectional analysis
}

\section{Faye Forsyth ${ }^{1}$, James Brimicombe ${ }^{1}$, Joseph Cheriyan' ${ }^{2}$, Duncan Edwards ${ }^{1}$, FD Richard Hobbs ${ }^{3}$, Navazh Jalaludeen ${ }^{2}$, Jonathan Mant ${ }^{1}$, Mark Pilling ${ }^{1}$, Rebekah Schiff ${ }^{4}$, Clare J Taylor ${ }^{3}$, M Justin Zaman ${ }^{3}$, Christi Deaton ${ }^{1,4 *}$, on behalf of the OPTIMISE HFpEF investigators and collaborators ${ }^{1}$}

${ }^{1}$ Department of Public Health and Primary Care, University of Cambridge School of Clinical Medicine, Cambridge, UK; ${ }^{2}$ Division of Experimental Medicine and Immunotherapeutics, Department of Medicine, University of Cambridge School of Clinical Medicine, Cambridge, UK; ${ }^{3}$ Nuffield Department of Primary Care Health Sciences, University of Oxford, Oxford, UK; ${ }^{4}$ Department of Ageing and Health, Guy's and St. Thomas' NHS Foundation Trust, London, UK

\begin{abstract}
Background: Many patients with heart failure with preserved ejection fraction (HFpEF) are undiagnosed, and UK general practice registers do not typically record heart failure (HF) subtype. Improvements in management of HFpEF is dependent on improved identification and characterisation of patients in primary care.
\end{abstract}

Aim: To describe a cohort of patients recruited from primary care with suspected HFpEF and compare patients in whom HFpEF was confirmed and refuted.

Design \& setting: Baseline data from a longitudinal cohort study of patients with suspected HFpEF recruited from primary care in two areas of England.

Method: A screening algorithm and review were used to find patients on HF registers without a record of reduced ejection fraction (EF). Baseline evaluation included cardiac, mental and physical function, clinical characteristics, and patient reported outcomes. Confirmation of HFpEF was clinically adjudicated by a cardiologist.

Results: In total, 93 (61\%) of 152 patients were confirmed HFpEF. The mean age of patients with HFpEF was 79 years, 46\% were female, $80 \%$ had hypertension, and $37 \%$ took $\geq 10$ medications. Patients with HFpEF were more likely to be obese, pre-frail or frail, report more dyspnoea and fatigue, were more functionally impaired, and less active than patients in whom HFpEF was refuted. Few had attended cardiac rehabilitation.

Conclusion: Patients with confirmed HFpEF had frequent multimorbidity, functional impairment, frailty, and polypharmacy. Although comorbid conditions were similar between people with and without HFpEF, the former had more obesity, symptoms, and worse physical function. These findings highlight the potential to optimise wellbeing through comorbidity management, medication rationalisation, rehabilitation, and supported self-management.

\section{How this fits in}

HFpEF is common (about half of all patients with HF) but the condition is often unrecognised and poorly managed. To the authors' knowledge, no previous studies have provided a detailed 
characterisation of patients with HFpEF within primary care HF registers. This study confirmed diagnosis and phenotyped a cohort of patients recruited from primary care with possible HFpEF, comparing patients in whom HFpEF was confirmed with patients in whom HFpEF was refuted. Patients with HFpEF were differentiated from patients not meeting HFpEF diagnostic criteria by higher levels of obesity, frailty and symptoms, and worse physical functioning. Self-management and self-monitoring of worsening signs and symptoms of HF were extremely limited in patients with HFpEF. Management of comorbidities in HFpEF is essential but complex. It needs to incorporate medication reviews, and increased use of non-pharmacological interventions such as self-management support and exercise training or cardiac rehabilitation. Polypharmacy could be decreased by better differentiation between patients with HFpEF and heart failure with reduced ejection fraction (HFrEF).

\section{Introduction}

HFpEF accounts for half of all HF and $70 \%$ of those with HF aged $>65$ years. ${ }^{1}$ Current evidence suggests HFpEF is driven by comorbid conditions, especially obesity, hypertension, diabetes, and kidney disease, leading to systemic inflammation and endothelial microvascular dysfunction. ${ }^{1,2}$ Despite its prevalence, HFpEF remains poorly diagnosed, managed, and researched. ${ }^{3-6}$ Under-recognition of HFpEF relates to lack of awareness and uncertainty regarding its pathophysiology, treatment, and diagnostic criteria. Pathways to HF diagnosis are variable, and limited knowledge of HFpEF and a lack of relevant echocardiographic information leads to under-identification in primary care. ${ }^{3-5,7}$

Most patients with HF are managed in primary care, especially those with HFpEF who may not be referred to specialists, or if referred not provided with a treatment plan. ${ }^{3,8}$ Evidence for effective pharmacological treatment specific to HFpEF is sparse. Current recommendations are to control comorbid conditions and use diuretics to manage volume overload. ${ }^{9}$ Lack of pharmacological treatment is thought to relate to patient heterogeneity, leading to interest in defining phenotypes that might respond to specific therapy. Phenotyping has been based on populations recruited into clinical trials with limited comorbidity or admitted for acute HF, and thus not representative of most patients in the community. ${ }^{10-13}$ Characterising patients with HFpEF in primary care is an essential step towards improving diagnosis and management, as well as recruiting into trials.

This analysis presents baseline data from a longitudinal observational study that is a component of the Optimising Management of Patients with Heart Failure with Preserved Ejection Fraction in Primary Care (OPTIMISE HFpEF) study. ${ }^{14}$ Patients were recruited based on a search of primary care $\mathrm{HF}$ registers for patients with no record of reduced ejection fraction. In this report, the baseline characteristics of those patients in the cohort who were confirmed as having HFpEF are described, and they are compared to patients in whom HFpEF was not confirmed.

\section{Method}

\section{Study design and setting}

Study participants were recruited from 30 general practices in two regions of England: East of England and Oxfordshire, Thames Valley. Practices were included from cities, towns, and semi-rural areas varying by Index of Multiple Deprivation (IMD) score from high deprivation to more affluent areas (IMD 2-9). Owing to slow accrual, patients were also recruited from an older persons' clinic in London and a HF service in Cambridgeshire receiving primary care referrals. The study is supported by an active patient advisory group, and patients were involved in development and analysis.

\section{Participants}

Patients with possible HFpEF were identified via an electronic medical record screening algorithm of HF registers in general practices and physical record screening in the outpatient clinics. The algorithm was designed to screen out patients with codes for left ventricular systolic dysfunction (LVSD) and cardiomyopathy. Patients identified in the electronic search were screened by GPs against study criteria. Exclusion criteria included an EF $<50 \%$, moderate to severe systolic dysfunction, significant cognitive impairment, or end-of-life care. Patients deemed eligible were invited to participate by letter from the practice. Those interested attended a baseline assessment where informed consent was obtained. 
Box 1 European Society of Cardiology criteria for diagnosis of HFpEF ${ }^{9}$

- Signs and symptoms of heart failure

- Ejection fraction $>50 \%$

- Elevated natriuretic peptides:

- NT-proBNP $>125 \mathrm{pg} / \mathrm{ml}$

- $\quad$ BNP $>35 \mathrm{pg} / \mathrm{ml}$

- Evidence of relevant structural heart disease and/or diastolic dysfunction:

- Left ventricular hypertrophy: left ventricular mass index $>115 \mathrm{~g} / \mathrm{m}^{2}$ for males and $>95 \mathrm{~g} / \mathrm{m}^{2}$ for females

- Increased left atrial volume index: $>34 \mathrm{ml} / \mathrm{m}^{2}$

- Early diastolic tissue velocity (e'mean septal-lateral $<9 \mathrm{~cm} / \mathrm{s}$ )

- Ratio between early mitral inflow velocity and mitral annular early diastolic velocity $\left(E / e^{\prime}>13\right)$

- E/A ratio $<1$ or $>2$

- Deceleration time of mitral valve early diastolic inflow $\mathrm{m} / \mathrm{s}$ (normal is $<240 \mathrm{~m} / \mathrm{s}$ )

- Isovolumetric relaxation time

$\mathrm{BNP}=$ brain natriuretic peptide. $\mathrm{HF} \mathrm{FEF}=$ heart failure with preserved ejection fraction. $\mathrm{NT}$-proBNP $=\mathrm{N}$-terminal pro $\mathrm{B}$-type natriuretic peptide.

HFpEF diagnosis was clinically adjudicated by a cardiologist based on a global evaluation of the available history of any heart failure symptoms, signs of HF, consideration of natriuretic peptide levels, and evidence of relevant structural heart disease and/or diastolic dysfunction on transthoracic echocardiogram (TTE), as per European Society of Cardiology (ESC) guidelines criteria at start of recruitment (Box 1). A more detailed discussion of the diagnostic process for the study is available elsewhere. ${ }^{15}$

\section{Variables}

Variables included: physical characteristics; past medical history and comorbidities; heart function (12lead electrocardiogram and transthoracic echocardiogram [TTE]); oedema assessment; breathlessness and fatigue (modified Borg); frailty assessment by Clinical Frailty Scale (CFS); Survey of Health, Ageing and Retirement in Europe Frailty Instrument (SHARE-FI); cognition assessment (Montreal Cognitive Assessment [MoCA]); physical functioning (6-minute walk distance, gait speed) and physical activity levels (7 -day accelerometer wear); laboratory testing (biochemistry, haematology, biomarkers); anxiety and depression (Hospital Anxiety and Depression Score [HADS]); HF quality of life (Kansas City Cardiomyopathy Questionnaire [KCCQ]); HF self-care (European Heart Failure Self-care Behaviour

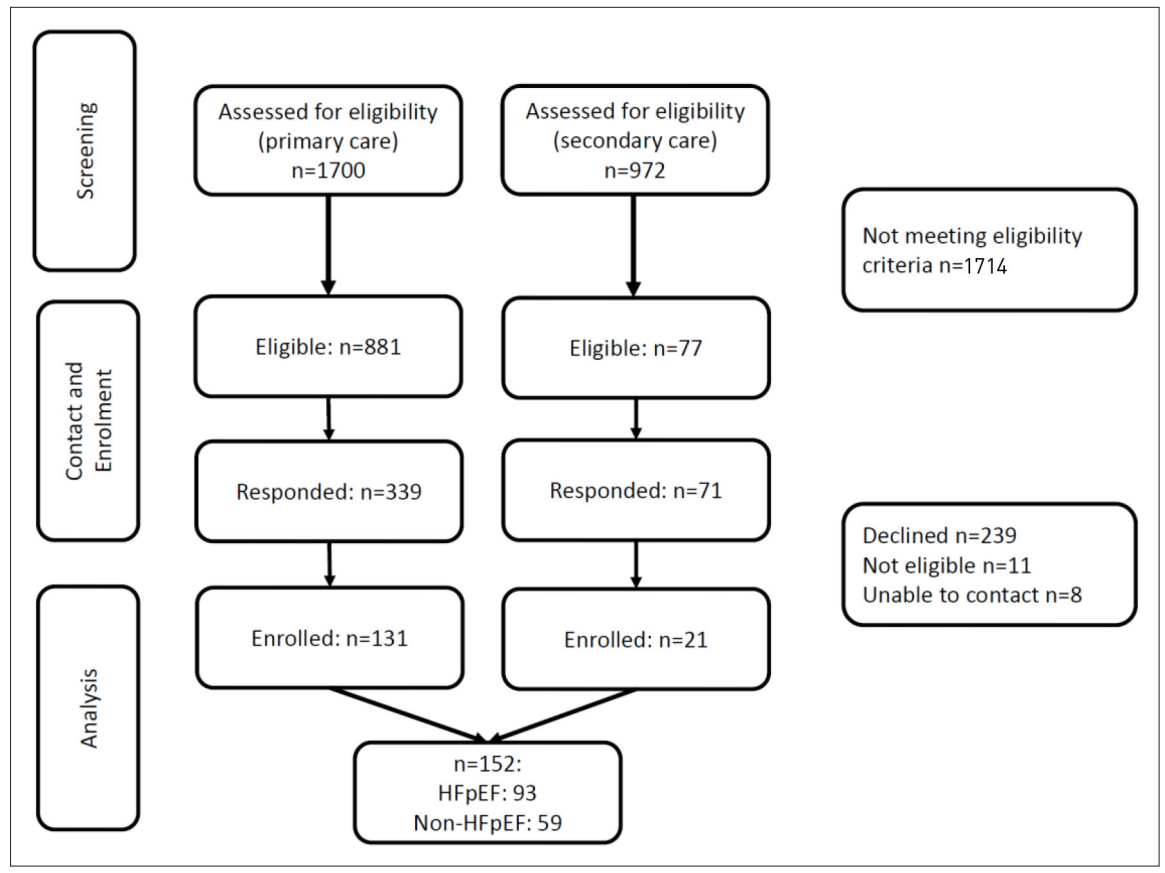

Figure 1 Patient flow chart. HFpFF = heart failure with preserved ejection fraction. 
[EHFScB] Scale); HF symptoms (Symptom Status Questionnaire - Heart Failure); and health-related quality of life (EQ-5D-5L). Validated assessments, standardised equipment, and a detailed manual of operations and procedures promoted consistency across sites.

\section{Sample size}

The target sample size was 270 recruited, with an estimated $25 \%$ not being confirmed as $\mathrm{HFpEF}^{16}$ to give a sample of 200 patients. It was anticipated that $40 \%$ of patients on HF registers would be identifiable as possible HFpEF. ${ }^{5}$

\section{Statistical analysis}

Patient characteristics and assessments were described using frequencies, measures of central tendency, and proportions as appropriate. Normality for continuous data were assessed using the Shapiro-Wilk test and Q-Q plots. Normally distributed data are presented as mean \pm standard deviation, non-parametric as median and interquartile range (25\%-75\%), and categorical data as absolute number and per cent. Descriptive statistics are presented for the cohort, and comparisons according to confirmed HFpEF versus non-HFpEF using $\chi^{2}$ for categorical variables and $t$-tests for normally distributed continuous data. Where data are missing, values are reported as such. Statistical analyses were performed using $\mathrm{R}$ (version 3.63) and IBM SPSS Statistics (version 27).

\section{Results}

In primary care, 52\% of patients on HF registers were considered eligible. Between July 2018 and November 2019, 152 patients were recruited, 16\% of those were eligible (Figure 1). Ninety-three (61\%) were clinically adjudicated to have HFpEF. Participants with HFpEF (Table 1) had a mean age of 79 years $( \pm 7.1), 46 \%$ were female, and $60 \%$ had a history of smoking. Mean Charlson Comorbidity Index $(\mathrm{CCl})$ was 4.8 , and the majority were overweight or obese. Functional impairment was evident by $6 \mathrm{MWD}$ and gait speed, and $63 \%$ had mild cognitive impairment. Over half were considered prefrail or frail, and $40 \%$ were considered sarcopaenic by grip strength and gait speed. Sixteen per cent reported occasional incontinence, and $4 \%$ were incontinent or had indwelling catheters.

Although the initial aim was to characterise and follow-up patients with HFpEF, the authors took the opportunity in this baseline analysis to compare patients confirmed as HFpEF with those not considered to have HFpEF. The non-HFpEF group primarily had a mixture of other HF diagnoses (for example, valvular heart disease, hypertrophic cardiomyopathy, and recovered EF), although the investigations were only intended to diagnose patients without HFpEF. When delineating by confirmation of HFpEF, patients with HFpEF were more likely to be pre-frail or frail, and have greater functional impairment based on 6MWD and gait speed. Patients with HFpEF were less physically active and spent more time in very low levels of activity compared with those not confirmed HFpEF. ${ }^{17}$ Patients with HFpEF walked $65 \mathrm{~m}$ less than the non-HFpEF group, and took $>2$ seconds longer to walk $10 \mathrm{~m}$. Sarcopaenia was more prevalent in the HFpEF versus non-HFpEF group (40\% versus $29 \%$, $P=0.176)$ (Table 1).

Laboratory tests were available for 131 (86\%) participants (Table 2). Values were not significantly different between groups, although an estimated glomerular filtration rate (eGFR) $<30 \mathrm{ml} / \mathrm{min}$ was more frequent in those with HFpEF compared with those without. Natriuretic peptides (NT-proBNP) levels were a median of $301 \mathrm{pg} / \mathrm{ml}$ (interquartile range [IQR] 73-1029) in the HFpEF group, and $332 \mathrm{pg} / \mathrm{ml}$ (IQR 147-1112) in those without HFpEF. A small number of patients (six with HFpEF) presented with NT-proBNP levels $>2000 \mathrm{pg} / \mathrm{ml}$. Twice as many patients with HFpEF had $\mathrm{HbA} 1 \mathrm{c}$ levels $>48 \mathrm{mmol} / \mathrm{I}$ than those without HFpEF (26\% versus $13 \%, P=0.085)$, and mean $\mathrm{HbA} 1 \mathrm{c}$ in 39 patients known to have diabetes was $56.4 \pm 16.7 \mathrm{mmol} / \mathrm{l}$.

Patient reported outcome measures (Table 3) showed no statistically significant differences in scores except for daytime dyspnoea and fatigue (worse in people with HFpEF). Pharmacological treatment (Table 4) did not differ significantly between groups, with both prescribed an average of eight medications. Approximately one-third of patients were on $\geq 10$ medications. Most patients were prescribed diuretics, and about half were on beta-blockers. In contrast to pharmacological treatment, cardiac rehabilitation was infrequent. 
Table 1 Characteristics of sample and by HFpEF diagnosis

\begin{tabular}{|c|c|c|c|c|c|}
\hline Characteristic & $n$ & $\begin{array}{c}\text { Total sample, } n= \\
152, \%^{\mathrm{a}}\end{array}$ & $\begin{array}{c}\text { Confirmed HFpEF, } \\
n=93, \% \text { a }\end{array}$ & $\begin{array}{c}\text { Non-HFpEF, } \\
n=59, \%^{\mathrm{a}}\end{array}$ & $\begin{array}{l}P \text { value for } \\
\text { comparison }^{\mathrm{b}}\end{array}$ \\
\hline Age, years, mean (SD) & 152 & $78(8.6)$ & $79(7.1)$ & $77(10.5)$ & 0.156 \\
\hline Sex, female & 152 & 40 & 46 & 29 & 0.039 \\
\hline LVEF, mean (SD) & 148 & $56.9(9.2)$ & $58.1(7.1)$ & $54.4(10.8)$ & 0.023 \\
\hline History of smoking & 152 & 67 & 60 & 79 & 0.015 \\
\hline $\mathrm{CCl}$, mean (SD) & 150 & $4.6(2.6)$ & $4.8(2.8)$ & $4.2(2.2)$ & 0.157 \\
\hline Hypertension & 150 & 80 & 80 & 77 & 0.636 \\
\hline Diabetes & 150 & 29 & 32 & 26 & 0.498 \\
\hline Chronic lung disease & 150 & 29 & 32 & 23 & 0.251 \\
\hline $\begin{array}{l}\text { Moderate to severe kidney } \\
\text { disease }\end{array}$ & 150 & 33 & 34 & 32 & 0.789 \\
\hline $\begin{array}{l}\text { Previous myocardial } \\
\text { infarction }\end{array}$ & 149 & 13 & 12 & 14 & 0.730 \\
\hline Peripheral vascular disease & 150 & 9 & 7 & 12 & 0.226 \\
\hline Previous stroke or TIA & 150 & 14 & 14 & 16 & 0.781 \\
\hline Cancer & 150 & 16 & 14 & 19 & 0.404 \\
\hline BMI, mean (SD) & 151 & $30.4(6.6)$ & $30.9(6.2)$ & $29.4(7.1)$ & 0.179 \\
\hline Overweight & 151 & 26 & 25 & 28 & 0.053 \\
\hline Obese & 151 & 50 & 57 & 39 & \\
\hline $\begin{array}{l}\text { Combined overweight or } \\
\text { obese }\end{array}$ & 151 & 76 & 82 & 67 & 0.036 \\
\hline NYHA class I & 152 & 22 & 17 & 31 & 0.118 \\
\hline NYHA class II & 152 & 57 & 62 & 48 & \\
\hline NYHA class III & 152 & 20 & 20 & 21 & \\
\hline Leg oedema & 152 & 45 & 46 & 43 & 0.707 \\
\hline Sinus rhythm & 152 & 45 & 50 & 39 & 0.435 \\
\hline Atrial fibrillation & 152 & 34 & 32 & 39 & \\
\hline Other & 152 & 21 & 19 & 23 & \\
\hline Heart rate, mean (SD) & 145 & $68(14)$ & $68(13)$ & $69(15)$ & 0.556 \\
\hline SBP, mean (SD) & 150 & $136(23)$ & $138(23)$ & $134(22)$ & 0.346 \\
\hline $\mathrm{SBP}>150$, mean $\%$ & 150 & 31 & 33 & 28 & 0.535 \\
\hline $\mathrm{DBP}$, mean (SD) & 150 & $77(12)$ & $77(12)$ & $78(11)$ & 0.577 \\
\hline $\mathrm{DBP}>90$, mean $\%$ & 150 & 16 & 15 & 18 & 0.643 \\
\hline Pulse pressure, mean (SD) & 150 & 59 (19) & $61(17)$ & $56(20)$ & 0.142 \\
\hline MoCA score, mean (SD) & 146 & $25.4(3.3)$ & $24.9(4.3)$ & $24.8(5.7)$ & 0.951 \\
\hline Mild cognitive impairment & 146 & 58 & 63 & 48 & 0.194 \\
\hline Pre-frail & 148 & 32 & 36 & 27 & 0.101 \\
\hline Frail & 148 & 22 & 26 & 18 & \\
\hline Combined pre-frail and frail & 148 & 54 & 63 & 45 & 0.033 \\
\hline $\begin{array}{l}6 \text {-minute walk distance, } \\
\text { mean (SD) }\end{array}$ & 117 & $296(127)$ & $273(123)$ & $338(125)$ & 0.007 \\
\hline $\begin{array}{l}\text { Time to walk } 10 \mathrm{~m} \text {, sec, mean } \\
\text { (SD) }\end{array}$ & 117 & $10.8(6.4)$ & $11.7(7.4)$ & $9.1(3.6)$ & 0.014 \\
\hline Gait speed, m/s, mean (SD) & 117 & $1.13(0.47)$ & $1.05(0.39)$ & $1.3(0.55)$ & 0.010 \\
\hline
\end{tabular}


Table 1 Continued

\begin{tabular}{|c|c|c|c|c|c|}
\hline Characteristic & $\mathbf{n}$ & $\begin{array}{c}\text { Total sample, } n= \\
152, \%^{\mathrm{a}}\end{array}$ & $\begin{array}{l}\text { Confirmed HFpEF, } \\
\qquad n=93, \%^{a}\end{array}$ & $\begin{array}{l}\text { Non-HFpEF, } \\
n=59, \%^{a}\end{array}$ & $\begin{array}{l}P \text { value for } \\
\text { comparison }^{b}\end{array}$ \\
\hline $\begin{array}{l}\text { Activity levels by median } \\
\text { daily vector magnitude (IQR) }\end{array}$ & 124 & $16.2(12.2-20.2)$ & $15.4(12.0-18.3)$ & $18.2(12.9-21.5)$ & 0.018 \\
\hline Sarcopaenia & 147 & 35 & 40 & 29 & 0.176 \\
\hline Occasional incontinence & 151 & 17 & 16 & 19 & 0.867 \\
\hline Incontinent or catheterised & 151 & 4 & 4 & 4 & \\
\hline \multicolumn{6}{|c|}{ Patients with known hypertension } \\
\hline SBP, mean (SD) & 122 & $142.3(22.2)$ & $144.5(22.8)$ & $138.9(20.9)$ & 0.203 \\
\hline $\mathrm{DBP}$, mean $(\mathrm{SD})$ & 122 & $79.8(10.9)$ & $79.3(11.1)$ & $80.6(10.8)$ & 0.529 \\
\hline Pulse pressure, mean (SD) & 122 & $62.6(18.7)$ & $65.2(18.6)$ & $58.3(18.4)$ & 0.059 \\
\hline
\end{tabular}

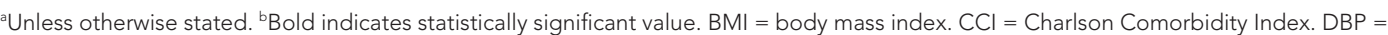
diastolic blood pressure. HFpEF = heart failure with preserved ejection fraction. IQR = interquartile range. LVEF = left ventricular ejection fraction. MoCA = Montreal Cognitive Assessment. NYHA = New York Heart Association. SBP = systolic blood pressure. SD = standard

deviation. TIA = transient ischaemic attack.

\section{Discussion}

\section{Summary}

In this cohort of patients recruited mainly from HF registers in primary care $(86 \%)$, the predominant characteristics of patients with HFpEF were a greater proportion of females, advanced age, and multimorbidity. Significant differences by group were found, as patients with HFpEF had more obesity, pre-frailty or frailty, functional impairment by 6MWD and gait speed, demonstrated lower levels of activity, and had greater likelihood of reporting symptoms, such as dyspnoea and fatigue, than those not confirmed HFpEF.

Table 2 Laboratory values by HFpEF diagnosis

\begin{tabular}{|c|c|c|c|c|c|}
\hline Parameter & $\mathbf{n}$ & $\begin{array}{c}\text { Total sample, } \\
n=152, \text { mean } \\
(S D)^{a}\end{array}$ & $\begin{array}{c}\text { Confirmed } \\
\text { HFpEF, } n=93, \\
\text { mean }(S D)^{\mathrm{a}}\end{array}$ & $\begin{array}{l}\text { Non-HFpEF, } n= \\
59, \text { mean }(\text { SD) }\end{array}$ & $\begin{array}{l}P \text { value for } \\
\text { comparison }\end{array}$ \\
\hline NT-proBNP, pg/ml, median (IQR) & 111 & $314(124-1055)$ & 301 (73-1029) & $332(147-1112)$ & 0.841 \\
\hline eGFR & 129 & $66(21)$ & $65(21)$ & $70(20)$ & 0.190 \\
\hline eGFR $<30, \%$ & 129 & 5 & 8 & 0 & 0.042 \\
\hline Random glucose, mmol/l & 120 & $6.8(4)$ & $6.9(3)$ & $6.7(4)$ & 0.797 \\
\hline Sodium, mmol// & 129 & $139(3)$ & $139(3)$ & $139(3)$ & 0.286 \\
\hline Potassium, mmol// & 128 & $4.2(0.5)$ & $4.2(0.4)$ & $4.2(0.5)$ & 0.401 \\
\hline Creatinine, $\mu \mathrm{mol} / / \mathrm{l}$ & 130 & $93(39)$ & $95(43)$ & $90(31)$ & 0.570 \\
\hline Urea, $\mathrm{mmol} / \mathrm{l}$ & 122 & $8.6(5)$ & $8.9(6)$ & $8.1(3)$ & 0.378 \\
\hline Haemoglobin, g/l & 131 & $131(17)$ & $130(15.5)$ & $135(19)$ & 0.130 \\
\hline Haematocrit, \% & 129 & $0.4(0.04)$ & $0.39(0.05)$ & $0.41(0.06)$ & 0.141 \\
\hline Platelets & 130 & $229(77)$ & $232(77)$ & $227(76)$ & 0.677 \\
\hline $\mathrm{HbA} 1 \mathrm{c}$ & 129 & $45(13)$ & $46(12)$ & $43(14)$ & 0.250 \\
\hline $\mathrm{HbA} 1 \mathrm{c}>48, \%$ & 129 & 22 & 26 & 13 & 0.085 \\
\hline HbA1c known diabetes & 39 & $56.4(16.7)$ & $56.5(14.2)$ & $56.2(22.6)$ & 0.955 \\
\hline
\end{tabular}

anless otherwise stated. eGFR = estimated glomerular filtration rate. $\mathrm{HbA} 1 \mathrm{c}=$ glycosylated haemoglobin $\mathrm{A} 1 \mathrm{c}$. $\mathrm{HFpEF}=$ heart failure with preserved ejection fraction. NT-proBNP $=\mathrm{N}$-terminal pro B-type natriuretic peptide. $\mathrm{SD}=$ standard deviation. 
Table 3 Patient reported measures by HFpEF diagnosis

\begin{tabular}{|c|c|c|c|}
\hline Patient reported outcome measures & HFpEF, $n=93, \%$ a & $\begin{array}{c}\text { Non-HFpEF, } n= \\
59, \% \text { a }\end{array}$ & $\begin{array}{c}P \text { value for com- } \\
\text { parison }\end{array}$ \\
\hline \multicolumn{4}{|l|}{ Kansas City Cardiomyopathy Questionnaire } \\
\hline Physical limitations, mean (SD) & $67(28)$ & $73(28)$ & 0.205 \\
\hline Quality of life, mean (SD) & $69(29)$ & $73(25)$ & 0.410 \\
\hline Symptom total, mean (SD) & $72(25)$ & $78(26)$ & 0.171 \\
\hline Clinical summary, mean (SD) & $70(24)$ & $77(25)$ & 0.118 \\
\hline Summary, mean (SD) & $71(25)$ & $74(25)$ & 0.374 \\
\hline \multicolumn{4}{|l|}{ Hospital Anxiety and Depression Scale } \\
\hline Depression mean (SD) & $7.6(2.3)$ & $7.2(2.5)$ & 0.236 \\
\hline Moderate-to-severe depressive symptoms & 8.9 & 6.9 & 0.810 \\
\hline Anxiety mean (SD) & $5.2(4.4)$ & $4.7(3.9)$ & 0.455 \\
\hline Moderate-to-severe anxiety symptoms & 11.2 & 12.1 & 0.904 \\
\hline \multicolumn{4}{|l|}{ EQ-5D-5L } \\
\hline Quality of life visual analogue scale, mean (SD) & $70(19)$ & $73(19)$ & 0.387 \\
\hline No problems with mobility & 29 & 36 & 0.815 \\
\hline No problems with self-care & 73 & 66 & 0.524 \\
\hline No problems with usual activities & 40 & 50 & 0.519 \\
\hline No pain or discomfort & 47 & 55 & 0.698 \\
\hline No anxiety or depression & 58 & 71 & 0.121 \\
\hline \multicolumn{4}{|c|}{ Symptom Status Questionnaire (reported symptoms) } \\
\hline Daytime dyspnoea & 63 & 46 & 0.035 \\
\hline Orthopnoea & 22 & 25 & 0.743 \\
\hline Fatigue or lack of energy & 81 & 61 & 0.012 \\
\hline Chest pain & 82 & 83 & 0.978 \\
\hline Difficulty sleeping & 47 & 46 & 0.901 \\
\hline Dizziness or loss of balance & 48 & 35 & 0.130 \\
\hline Total score, mean (SD) & $24.4(18.4)$ & $22.3(20.5)$ & 0.503 \\
\hline \multicolumn{4}{|l|}{ EHFScB } \\
\hline Total score, mean (SD) & $46.5(21.2)$ & $43.5(22.2)$ & 0.426 \\
\hline
\end{tabular}

Responded 'do not agree at all' on some individual items on EHFScB Scale

\begin{tabular}{llll}
\hline I weigh myself every day & 61 & 68 & 0.475 \\
\hline $\begin{array}{l}\text { If my shortness of breath increases, I contact my } \\
\text { doctor or nurse }\end{array}$ & 48 & 39 & 0.418 \\
\hline $\begin{array}{l}\text { If my feet or legs become more swollen than } \\
\text { usual I contact my doctor or nurse }\end{array}$ & 41 & 46 & 0.930 \\
$\begin{array}{l}\text { If I gain } 2 \text { kg in } 1 \text { week I contact my doctor or } \\
\text { nurse }\end{array}$ & 72 & 70 & 0.937
\end{tabular}

aUnless otherwise stated. EHFSCB = European Heart Failure Self-care Behaviours. EQ-5D-5L = EuroOoL - 5 dimensions - 5 levels. HFpEF = heart failure with preserved ejection fraction. SD = standard deviation. 
Table 4 Pharmacological treatment by HFpEF diagnosis

\begin{tabular}{|c|c|c|c|}
\hline Pharmacological agent & $\begin{array}{c}\text { HFpEF, } \\
n=93, \%^{a}\end{array}$ & $\begin{array}{l}\text { Non-HFpEF, } \\
n=59, \%{ }^{\text {a }}\end{array}$ & $P$ value for comparison \\
\hline Prescribed medications, mean (SD) & $8.3(4.0)$ & $7.8(3.9)$ & 0.454 \\
\hline$\geq 10$ medications & 37 & 30 & 0.398 \\
\hline ACEI & 34 & 37 & 0.698 \\
\hline ARB & 30 & 32 & 0.840 \\
\hline ARNI & 1 & 2 & 0.743 \\
\hline MRA & 12 & 18 & 0.355 \\
\hline Beta-blockers & 48 & 54 & 0.475 \\
\hline Calcium channel blockers & 32 & 40 & 0.315 \\
\hline Loop diuretics & 57 & 51 & 0.456 \\
\hline Any diuretic & 65 & 61 & 0.673 \\
\hline Digoxin & 16 & 22 & 0.334 \\
\hline Statins & 58 & 63 & 0.552 \\
\hline Aspirin & 21 & 28 & 0.316 \\
\hline Other antiplatelet & 7 & 5 & 0.729 \\
\hline Anticoagulation & 51 & 65 & 0.100 \\
\hline Anticoagulation if $\mathrm{AF}^{\mathrm{b}}$ & 96 & 91 & 0.409 \\
\hline Antidepressants & 16 & 9 & 0.232 \\
\hline Anti-anaemia drugs & 14 & 5 & 0.111 \\
\hline Uric acid-related drugs & 19 & 18 & 0.813 \\
\hline NSAIDs & 2 & 2 & 0.845 \\
\hline
\end{tabular}

Patients with diabetes $(n=44)$

\begin{tabular}{lccc}
\hline Insulin & 25 & 40 & 0.307 \\
\hline Biguanides & 48 & 47 & 0.927 \\
\hline Sulfonylureas & 15 & 20 & 0.666 \\
\hline SGLT2 inhibitors & 7 & 0 & 0.535 \\
\hline DPP4 inhibitors & 17 & 20 & 0.822 \\
\hline Non-pharmacologic management & & & \\
\hline Attended CR in past & 13 & 16 & 0.640 \\
\hline Currently attending CR & 3 & 0 & 0.168 \\
\hline
\end{tabular}

anless otherwise stated. ${ }^{b} n=48 . \mathrm{ACEl}=$ angiotensin-converting enzyme inhibitor. $\mathrm{AF}=$ atrial fibrillation. $\mathrm{ARB}=$ angiotensin receptor blocker. $\mathrm{ARNI}=$ angiotensin receptor-neprilysin inhibitor. $\mathrm{CR}=$ cardiac rehabilitation. $\mathrm{DPP} 4=$ dipeptidyl peptidase-4. HFpEF = heart failure with preserved ejection fraction. MRA = mineralocorticoid receptor antagonist. NSAIDs = non-steroidal anti-inflammatory drugs. SD = standard deviation. SGLT2 = sodium glucose co-transporter-2.

As might be expected in an older multimorbid sample, patients were taking multiple medications. Sixty-five per cent of patients with HFpEF were taking diuretics, but many presented with signs and symptoms of volume overload such as peripheral oedema. Although few abnormalities were found in laboratory values, HbA1c levels in patients with diabetes indicated that glycaemic control was less than optimal. Findings on the EHFScB indicated that few patients with HFpEF agreed with statements that they regularly performed actions recommended for self-management such as monitoring weight gain or notifying a healthcare provider for signs and symptoms of worsening HF. Patients did not 
report high levels of depression or anxiety symptoms, and quality-of-life scores were moderately high on both the KCCQ and EQ-5D-5L visual analogue scale.

\section{Strengths and limitations}

This study presents a well-phenotyped cohort of patients with HFpEF recruited mainly from primary care practices in two regions in England, indicating the challenges and problems faced. Recruitment was slow, and likely limited by focusing on patients on practice HF registers, so patients not yet diagnosed with HF or with HF not added to the register were excluded. Future studies may find more patients by searching the practice adult population for those on diuretics or combinations of medications used for HF. ${ }^{18}$ Over half of the eligible sample did not respond to the study invitation, and $58 \%$ of those responding declined participation. Information about non-responders or those declining was not collected, but it is plausible that some may have had poorer health or not thought the study was relevant to them. Limited recruitment may have introduced bias in the sample; however, it is notable that the sample was older, multimorbid, functionally impaired, and came from both low and high areas of deprivation. The sample was limited by a high proportion of patients not confirmed as HFpEF. Confirmation of HFpEF was clinically adjudicated using symptoms, signs, NT-proBNP, and echocardiogram data, following European Society of Cardiology (ESC) guidelines criteria. ${ }^{9}$ Future studies may include additional testing to determine diagnosis.

\section{Comparison with existing literature}

The prevalence of comorbidities has been reported to be higher in HFpEF than HFrEF, consistent with the idea that comorbid conditions drive the inflammatory response leading to HFpEF. ${ }^{16}$ The older patients with HFpEF with multiple comorbid conditions, such as obesity, hypertension, diabetes, and kidney disease, has been described as 'garden variety' HFpEF, indicating that this is a frequent phenotype encountered in clinical practice. ${ }^{1}$ However, this common phenotype contrasts with HF clinical trials where limited reporting of comorbid conditions and low prevalence of obesity and multimorbidity is usual in recruited patients with HFpEF. ${ }^{13}$ Studies have attempted to delineate patients into distinct phenotypes based on clinical and diagnostic characteristics using patient samples from secondary care centres and clinical trials. ${ }^{10-12}$ Currently there is no agreement on distinct phenogroups, and others have called for simpler designations using single characteristics such as sex, obesity, and atrial fibrillation. ${ }^{19,20}$ This analysis fills a gap in the literature by detailing the characteristics of the prevalent patient who is older and multimorbid with HFpEF in primary care, revealing areas of need in their management.

Multiple studies have shown a greater prevalence of women among populations with HFpEF, although it is unclear whether this is related to higher survival rates of women at older ages, or factors such as the stronger relationship between obesity and incident HFpEF among women compared with men. ${ }^{21}$ Overweight and obesity is highly prevalent in patients with HFpEF (up to $80 \%$ ), as is frailty. ${ }^{20,22,23}$ A recent analysis of 4605 older patients (mean age 80.3 years) with HFpEF hospitalisation found that $41 \%$ had frailty, and that frailty was the most important predictor of re-hospitalisation, and second (after age) for mortality. ${ }^{23}$

Exercise intolerance in HFpEF is owing to both cardiac and peripheral factors, with pro-inflammatory factors, fatty infiltration, and impaired oxidative metabolism leading to decreased muscle strength. ${ }^{24}$ The average 6MWD difference between groups was $65 \mathrm{~m}$. A recent meta-analysis found each $50 \mathrm{~m}$ 6MWD reduction was significantly associated with increased risk of all-cause mortality, readmission rates, and combined death or readmission. ${ }^{25}$ Although all patients had low activity levels, the average vector magnitude was lower in those with HFpEF compared with patients without HFpEF, and less than in a UK Biobank sample of patients with HF and in another study of HFpEF. ${ }^{17,26,27}$

Somewhat surprisingly, despite symptoms and limited functional status, quality-of-life scores were moderately high. The developers of the KCCQ define scores from 50 to 74 as fair to good health status, and $\geq 75$ as good to excellent. ${ }^{28}$ The overall score on the EHFScB Scale was low compared with a sample of 1192 patients with either HFpEF or HFrEF (mean score 58.3, mean age 72 years, mean EF $45 \%)$, indicating fewer self-care behaviours among the cohort in the present study. ${ }^{29}$ 


\section{Implications for practice}

The study demonstrates that multimorbidity, polypharmacy, obesity, pre-frailty and frailty, poor physical function, low activity levels, and symptoms are prevalent in patients with HFpEF and present key management challenges. Patients with HFpEF often sit outside of specialist HF services in the UK owing to commissioning restrictions, and primary care therefore takes the lead in managing patients. ${ }^{8,30}$ Current recommendations to manage comorbid conditions and to use diuretics ${ }^{9}$ are not trivial given the number of co-existing conditions, detrimental effects of polypharmacy, and challenges of fluid balance in older adults with renal and functional impairment.

Implications for primary care practice begin with the identification of patients with HFpEF, which likely needs specialist support, ${ }^{15}$ but is important in ensuring appropriate treatment. For example, a decrease in polypharmacy in HFpEF could be enhanced by differentiation of HFpEF from HFrEF. Medications indicated for HFrEF should not be prescribed unless there is another indication (for example, angiotensin-converting enzyme inhibitor for blood pressure control), as they do not exert the same protective effect in HFpEF. ${ }^{1}$ Medication reviews in primary care provide the opportunity to consider the necessity for specific medications.

Over half of the patients in both groups were prescribed diuretics, which often limit their ability and willingness to leave the house. The challenge of managing diuresis is further complicated if patients have incontinence, as reported by almost $20 \%$ of patients in the sample. Managing fluid balance also requires consideration of patient behaviours and support to enable patients to monitor signs and symptoms, limit fluid and excessive salt intake if appropriate, and know when to contact a healthcare provider. ${ }^{9}$ Scores on the EHFScB indicated that many patients did not practice behaviours related to self-management. Teaching and supporting self-management should be a component of HF reviews, and all providers need to facilitate this partnership with patients.

Interventions to improve general health status, such as physical activity, dietary enhancement, and management of breathlessness, should be introduced. Exercise training or bespoke cardiac rehabilitation could be developed and commissioned given the evidence of benefit. ${ }^{27,31}$ Home-based targeted rehabilitation, such as in the REACH-HFpEF pilot study, ${ }^{27}$ may improve patient and carer outcomes and be key to ensuring patient participation. The Rehab-HF trial demonstrated that patients who were recently hospitalised and very frail with $\mathrm{HF}$ benefit from rehabilitation. ${ }^{32}$

New therapies to treat HFpEF may be added to current medication regimens in the future. Indications from recent studies are that medications such as sodium-glucose co-transporter-2 (SGLT2) inhibitors, spironolactone, and sacubitril with valsartan may be effective, even if in specific subgroups. ${ }^{1}$ The American Heart Association and American College of Cardiology have made a limited recommendation for the use of spironolactone in some patients with HFpEF. ${ }^{33}$

Patients recruited from primary care with confirmed HFpEF demonstrate marked impairment across a range of domains including multimorbidity, functional impairment, and frailty. These findings highlight the need to recognise and record HFpEF as a diagnosis, which would enable clinicians to identify patients and work together to optimise wellbeing through comorbidity management, medication rationalisation, rehabilitation, and self-management.

\section{Funding}

This work was supported by the National Institute for Health Research School for Primary Care Research (NIHR SPCR) (grant number: 384), the NIHR Cambridge Biomedical Research Centre (reference number: BRC-1215-20014), and conducted at and supported by the NIHR Cambridge Clinical Research Facility. The views expressed are those of the authors and not necessarily those of the NIHR, the NHS, or the Department of Health and Social Care. The study sponsors were not involved in any aspect of the study including study design, data collection, data analysis, and interpretation of data. Clare J Taylor is funded by an NIHR academic clinical lectureship. FD Richard Hobbs acknowledges part-funding from the NIHR SPCR, the NIHR Collaboration for Leadership in Health Research and Care Oxford, the NIHR Oxford Biomedical Research Centre, and the NIHR Oxford Medtech and In-Vitro Diagnostics Co-operative. Christi Deaton has funding from NIHR, NIHR SPCR, and Addenbrookes Charitable Trust. Jonathan Mant is an NIHR Senior Investigator.

Ethical approval

Granted by the London-Surrey Research Ethics Committee (reference number: 17/LO/2136). 
Trial registration number

ClinicalTrials. gov (reference number: NCT03617848).

\section{Provenance}

Freely submitted; externally peer reviewed.

\section{Acknowledgements}

The authors would like to acknowledge the patients and healthcare providers who supported this study.

\section{Competing interests}

Clare J Taylor reports personal fees from Vifor and Novartis, and non-financial support from Roche outside the submitted work. The remaining authors have declared no competing interests.

\section{Contributors}

Mollika Chakravorty, Sophie Maclachlan, Edward Kane, Jessica Odone, Natasha Thorley, Susana Borja-Boluda, Ian Wellwood, Emma Sowden, Thomas Blakeman, Carolyn Chew-Graham, Muhammed Hossain, John Sharpley, Brain Gordon, Joanna Taffe, Aaron Long, Affan Aziz, Hannah Swayze, Heather Rutter, Chris Schramm, Sine MacDonald, Helena Papworth, Julie Smith, Craig Needs, David Cronk, Chris Newark, Duncan Blake, Alistair Brown, Amman Basuita, Emma Gayton, Victoria Glover, Robin Fox, Jonathan Crawshaw, Helen Ashdown, Christine A'Court, Rachael Ayerst, Basilio Hernandez-Diaz, Kyle Knox, Nick Wooding, Shamila Wanninayake, Christopher Keast, Adam Jones, Katherine Brown, Matthew Gaw, Nick Thomas, Sharon Dixon, Elisabetta Angeleri-Rand.

\section{References}

1. Borlaug BA. Evaluation and management of heart failure with preserved ejection fraction. Nat Rev Cardiol 2020; 17(9): 559-573. DOI: https://doi.org/10.1038/s41569-020-0363-2

2. Paulus WJ, Tschöpe C. A novel paradigm for heart failure with preserved ejection fraction: comorbidities drive myocardial dysfunction and remodeling through coronary microvascular endothelial inflammation. J Am Coll Cardiol 2013; 62(4): 263-271. DOI: https://doi.org/10.1016/j.jacc.2013.02.092

3. Sowden $\mathrm{E}$, Hossain M, Chew-Graham C, et al. Understanding the management of heart failure with preserved ejection fraction: a qualitative multiperspective study. Br J Gen Pract 2020; 70(701): e880-e889. DOI: https://doi. org/10.3399/bjgp20X713477

4. Conrad N, Judge A, Canoy D, et al. Diagnostic tests, drug prescriptions, and follow-up patterns after incident heart failure: a cohort study of 93,000 UK patients. PLoS Med 2019; 16(5): e1002805. DOI: https://doi.org/10.1371/ journal.pmed. 1002805

5. Deaton C, Edwards D, Malyon A, S Zaman MJ. The tip of the iceberg: finding patients with heart failure with preserved ejection fraction in primary care. An observational study. BJGP Open 2018; 2(3): bjgpopen18X101606. DOI: https://doi.org/10.3399/bjgpopen18X101606

6. Ho JE, Redfield MM, Lewis GD, et al. Deliberating the diagnostic dilemma of heart failure with preserved ejection fraction. Circulation 2020; 142(18): 1770-1780. DOI: https://doi.org/10.1161/CIRCULATIONAHA.119.041818

7. Bottle $A$, Kim $D$, Aylin $P$, et al. Routes to diagnosis of heart failure: observational study using linked data in England. Heart 2018; 104(7): 600-605. DOI: https://doi.org/10.1136/heartjnl-2017-312183

8. Zheng A, Cowan E, Mach L, et al. Characteristics and outcomes of patients with suspected heart failure referred in line with National Institute for Health and Care Excellence guidance. Heart 2020; 106(20): 1579-1585. DOI: https:// doi.org/10.1136/heartjnl-2019-316511

9. Ponikowski P, Voors AA, Anker SD, et al. 2016 ESC Guidelines for the diagnosis and treatment of acute and chronic heart failure: The Task Force for the diagnosis and treatment of acute and chronic heart failure of the European Society of Cardiology (ESC). Developed with the special contribution of the Heart Failure Association (HFA) of the ESC. Eur Heart J 2016; 37(27): 2129-2200. DOI: https://doi.org/10.1093/eurheartj/ehw128

10. Kao DP, Lewsey JD, Anand IS, et al. Characterization of subgroups of heart failure patients with preserved ejection fraction with possible implications for prognosis and treatment response. Eur J Heart Fail 2015; 17(9): 925-935. DOI: https://doi.org/10.1002/ejhf.327

11. Shah SJ, Katz DH, Selvaraj S, et al. Phenomapping for novel classification of heart failure with preserved ejection fraction. Circulation 2015; 131(3): 269-279. DOI: https://doi.org/10.1161/CIRCULATIONAHA.114.010637

12. Segar MW, Patel KV, Ayers $C$, et al. Phenomapping of patients with heart failure with preserved ejection fraction using machine learning-based unsupervised cluster analysis. Eur J Heart Fail 2020; 22(1): 148-158. DOI: https://doi. org/10.1002/ejhf.1621

13. Khan MS, Samman Tahhan A, Vaduganathan $M$, et al. Trends in prevalence of comorbidities in heart failure clinical trials. Eur J Heart Fail 2020; 22(6): 1032-1042. DOI: https://doi.org/10.1002/ejhf.1818 
14. Forsyth F, Mant J, Taylor CJ, et al. Optimising Management of Patients with Heart Failure with Preserved Ejection Fraction in Primary Care (OPTIMISE-HFpEF): rationale and protocol for a multi-method study. BJGP Open 2019; 3(4): bjgpopen19X101675. DOI: https://doi.org/10.3399/bjgpopen19X101675

15. Forsyth F, Brimicombe J, Cheriyan J, et al. Diagnosis of patients with heart failure with preserved ejection fraction in primary care: cohort study. ESC Heart Fail 2021; DOI: https://doi.org/10.1002/ehf2.13612

16. Dalos D, Mascherbauer J, Zotter-Tufaro C, et al. Functional status, pulmonary artery pressure, and clinical outcomes in heart failure with preserved ejection fraction. J Am Coll Cardiol 2016; 68(2): 189-199. DOI: https://doi.org/10. 1016/j.jacc.2016.04.052

17. Lin H, Hartley P, Forsyth F, et al. Clinical and demographic correlates of accelerometer-measured physical activity in participants enrolled in the OPTIMISE HFpEF study. Eur J Cardiovasc Nurs 2021: zvab028 DOI: https://doi.org/ 10.1093/eurjen/zvab028

18. Cuthbert JJ, Gopal J, Crundall-Goode A, Clark AL. Are there patients missing from community heart failure registers? An audit of clinical practice. Eur J Prev Cardiol 2019; 26(3): 291-298. DOI: https://doi.org/10.1177/ 2047487318810839

19. Pedrotty DM, Jessup M. "Frailty, thy name is woman": syndrome of women with heart failure with preserved ejection fraction. Circ Cardiovasc Qual Outcomes 2015; 8(2 Suppl 1): S48-S51. DOI: https://doi.org/10.1161/ CIRCOUTCOMES.115.001700

20. Obokata M, Reddy YNV, Pislaru SV, et al. Evidence supporting the existence of a distinct obese phenotype of heart failure with preserved ejection fraction. Circulation 2017; 136(1): 6-19. DOI: https://doi.org/10.1161/ CIRCULATIONAHA.116.026807

21. Groenewegen A, Rutten FH, Mosterd A, Hoes AW. Epidemiology of heart failure. Eur J Heart Fail 2020; 22(8): 1342-1356. DOI: https://doi.org/10.1002/ejhf.1858

22. Kitzman DW, Shah SJ. The HFpEF obesity phenotype: the elephant in the room. J Am Coll Cardiol 2016; 68(2): 200-203. DOI: https://doi.org/10.1016/j.jacc.2016.05.019

23. Goyal $P$, Yum B, Navid $P$, et al. Frailty and post-hospitalization outcomes in patients with heart failure with preserved ejection fraction. Am J Cardiol 2021; 148: 84-93. DOI: https://doi.org/10.1016/j.amjcard.2021.02.019

24. Upadhya B, Haykowsky MJ, Eggebeen J, Kitzman DW. Exercise intolerance in heart failure with preserved ejection fraction: more than a heart problem. J Geriatr Cardiol 2015; 12(3): 294-304. DOI: https://doi.org/10.11909/j.issn. 1671-5411.2015.03.013

25. Fan Y, Gu X, Zhang H. Prognostic value of six-minute walk distance in patients with heart failure: a meta-analysis. Eur J Prev Cardiol 2019; 26(6): 664-667. DOI: https://doi.org/10.1177/2047487318797400

26. Barker J, Smith Byrne K, Doherty A, et al. Physical activity of UK adults with chronic disease: cross-sectional analysis of accelerometer-measured physical activity in 96706 UK Biobank participants. Int J Epidemiol 2019; 48(4): 1167-1174. DOI: https://doi.org/10.1093/ije/dyz148

27. Lang CC, Smith K, Wingham J, et al. A randomised controlled trial of a facilitated home-based rehabilitation intervention in patients with heart failure with preserved ejection fraction and their caregivers: the REACH-HFpEF pilot study. BMJ Open 2018; 8(4): e019649. DOI: https://doi.org/10.1136/bmjopen-2017-019649

28. Spertus JA, Jones PG, Sandhu AT, Arnold SV. Interpreting the Kansas City Cardiomyopathy Questionnaire in clinical trials and clinical care: JACC state-of-the-art review. J Am Coll Cardiol 2020; 76(20): 2379-2390. DOI: https://doi.org/10.1016/j.jacc.2020.09.542

29. Vellone E, Jaarsma T, Strömberg A, et al. The European Heart Failure Self-care Behaviour Scale: new insights into factorial structure, reliability, precision and scoring procedure. Patient Educ Couns 2014; 94(1): 97-102. DOI: https://doi.org/10.1016/j.pec.2013.09.014

30. Masters J, Barton C, Blue L, Welstand J. Increasing the heart failure nursing workforce: recommendations by the British Society for Heart Failure Nurse Forum. British Journal of Cardiac Nursing 2019; 14(11): 1-12. DOI: https:// doi.org/10.12968/bjca.2019.0109

31. Pandey A, Parashar A, Kumbhani D, et al. Exercise training in patients with heart failure and preserved ejection fraction: meta-analysis of randomized control trials. Circ Heart Fail 2015; 8(1): 33-40. DOI: https://doi.org/10.1161/ CIRCHEARTFAILURE.114.001615

32. Kitzman DW, Whellan DJ, Duncan $P$, et al. Physical rehabilitation for older patients hospitalized for heart failure. $N$ Engl J Med 2021; 385(3): 203-216. DOI: https://doi.org/10.1056/NEJMoa2026141

33. Yancy CW, Jessup M, Bozkurt B, et al. 2017 ACC/AHA/HFSA focused update of the 2013 ACCF/AHA guideline for the management of heart failure: a report of the American College of Cardiology/American Heart Association Task Force on clinical practice guidelines and the Heart Failure Society of America. Circulation 2017; 136(6): e137-e161. DOI: https://doi.org/10.1161/CIR.0000000000000509 